\title{
The WIGGUM gene is required for proper regulation of floral meristem size in
} \section{Arabidopsis}

\author{
Mark P. Running ${ }^{\star}$, Jennifer C. Fletcher and Elliot M. Meyerowitz ${ }^{\dagger}$ \\ California Institute of Technology, 1200 East California Boulevard, Pasadena, CA 91125, USA \\ *Present address: University of California Berkeley, USDA-ARS Plant Gene Expression Center, 800 Buchanon St, Albany, CA 94710, USA \\ †Author for correspondence (e-mail: meyerow@cco.caltech.edu) \\ Accepted 8 May; published on WWW 23 June 1998
}

\section{SUMMARY}

The study of cell division control within developing tissues is central to understanding the processes of pattern formation. The floral meristem of angiosperms gives rise to floral organs in a particular number and pattern. Despite its critical role, little is known about how cell division is controlled in the floral meristem, and few genes involved have been identified. We describe the phenotypic effects of mutations in WIGGUM, a gene required for control of cell proliferation in the floral and apical meristem of Arabidopsis thaliana. wiggum flowers contain more organs, especially sepals and petals, than found in wild-type flowers. This organ number phenotype correlates with specific size changes in the early floral meristem, preceding organ initiation. Genetic studies suggest that WIGGUM acts on a similar process but in a separate pathway than the CLAVATA1 and CLAVATA3 genes in meristem size regulation, and reveal interactions with other genes affecting meristem structure and identity. Analysis of double mutant phenotypes also reveals a role for WIGGUM in apical meristem function. We propose that WIGGUM plays a role in restricting cell division relative to cellular differentiation in specific regions of the apical and floral meristems.

Key words: Arabidopsis, WIGGUM (WIG), Floral meristem, Flower development, Pattern formation

\section{INTRODUCTION}

The flower meristem in angiosperms gives rise to floral organs in numbers and patterns characteristic of an individual species. In Arabidopsis thaliana, floral meristems initiate four concentric rings, or whorls, of organs, with 4 sepals in the first (outer) whorl, 4 petals in the second whorl, 6 stamens in the third whorl, and 2 fused carpels in the central gynoecium. The organs arise in defined positions as well, presumably due to stereotypic changes in cell division patterns at organ initiation sites that give rise to organ primordia, which then undergo further cell divisions and cell differentiation to form the mature organ.

The mechanisms by which the sites of organ initiation in the floral meristem are determined is unknown. One recent approach to studying this problem has been to isolate mutations that change the number and position of organs. Mutations in the CLAVATAI (CLVI) and CLAVATA3 (CLV3) genes increase organ number in all 4 floral whorls, especially the inner whorls, as well as increasing the total number of whorls (Leyser and Furner, 1992; Clark et al., 1993, 1995; Crone and Lord, 1993; Alvarez and Smyth, 1994). Mutations in the PERIANTHIA $(P A N)$ gene cause an increase in sepal and petal number and a slight decrease in stamen number (Running and Meyerowitz, 1996). Mutations in the FASCIATA1 (FASI) and FASCIATA2 (FAS2) genes show a variable organ number change in the first three whorls of the flower (Leyser and Furner, 1992). Mutations in TOUSLED (TSL; Roe et al., 1993, 1997), REVOLUTA (REV; Talbert et al., 1995), SHOOT MERISTEMLESS (STM; Barton and Poethig, 1993; Clark et al., 1996; Endrizzi et al., 1996), AINTEGUMENTA (ANT; Elliot et al., 1996; Klucher et al., 1996) and WUSCHEL (WUS; Laux et al., 1996) lead to decreased organ numbers in all 4 whorls.

In $c l v 1$ and $c l v 3$ mutants, the increase in organ and whorl number is correlated with specific changes in floral meristem size at the time of organ initiation (Clark et al., 1993, 1995; Alvarez and Smyth, 1994). Defects in the apical and/or floral meristem size or structure have also been reported in fas 1, fas 2, stm and wus mutants (Leyser and Furner, 1992; Barton and Poethig, 1993; Clark et al., 1996; Laux et al., 1996; Endrizzi et al., 1996).

In this paper we describe the effects of mutations in $W I G G U M(W I G)$, a gene required for maintenance of apical and floral meristem structure, floral organ number, and organ patterning. wig mutant plants show an increase in organ number similar to those seen in pan mutants, with extra sepals and petals, and some effects on stamen and carpel number as well. However, detailed studies of the wig mutant phenotype, as well as analysis of double mutant combinations of wig with other mutants, suggest that $W I G$ acts in manners distinct from those of previously described genes, and uncovers a novel 
mechanism for cell division control in apical and floral meristems.

\section{MATERIALS AND METHODS}

Seeds were sown at least $2 \mathrm{~cm}$ apart on an approximately 1:1:1 mix of soil:vermiculite:perlite, imbibed at $4^{\circ} \mathrm{C}$ for $4-5$ days, then placed under 600 foot-candles of continuous cool-white fluorescent light at $23^{\circ} \mathrm{C}$. Plants were given Miracle-Gro fertilizer (1:1500 dilution) at approximately 2 week intervals. For tests of the germination rate and time, seeds that were freshly harvested and dried for 2 weeks were sown on water-saturated Whatman no. 3 filter paper in closed Petri dishes, imbibed at $4^{\circ} \mathrm{C}$ for 5 days, and placed under the same light conditions as above.

All mutant alleles of wig were isolated using ethyl methanesulphonate (EMS) as a mutagen. wig-1 was isolated in the Landsberg erecta (L-er) ecotype and was backcrossed to wild-type Ler two times before analysis. wig-2 and wig-3 were isolated in the Nossen (No-0) ecotype and were backcrossed to L-er two times before analysis. All wig plants were homozygous for the erecta mutation. The hanaba taranu and snowball mutations arose independently in a population of Agrobacterium tumefaciens-mediated transformation mutants of ecotype Wassilewskija (Ws; Feldmann, 1992). All other mutant lines have been previously described.

$\mathrm{F}_{2}$ plants from a cross of wig-1 to the wild-type Columbia (Col-2) ecotype were used in determining the chromosomal map position of WIG relative to simple sequence length polymorphisms described by Bell and Ecker (1994). Two loci on chromosome 5 showed linkage: nga76 was found to be $12.2 \mathrm{cM}$ from wig-1, and nga139 was found to be $25 \mathrm{cM}$ from wig-1. Linkage was also noted with the visible markers fas 2 (Leyser and Furner, 1992) and pinhead (pnh; McConnell and Barton, 1995).

Scanning electron microscopy was performed as described by Bowman et al. (1989). Confocal laser scanning microscopy was performed as described by Running et al. (1995).

\section{RESULTS}

\section{wiggum affects floral organ number}

Mutants in the WIGGUM gene were isolated on the basis of their increased floral organ number compared to wild type (Table 1; Fig. 1). All four whorls are affected, though petal and sepal number is increased the most. Organ number is more variable than in wild type: most flowers in wig- 1 plants have 4-6 sepals, 4-6 petals, and 5-7 stamens (Table 1), and occasionally flowers with up to 8 of one type of organ are seen. Variation in sepal, petal and carpel number is rarely seen in wild-type L-er (Smyth et al., 1990). The organ number increase is greatest in the basal flowers, and decreases in apical flowers to numbers closer to wild type, except that carpel number remains stable. wig-3 floral organ number is similar to that of wig-1, while wig-2 has more sepals and petals (Table 1). All 3 alleles are recessive.

The number of floral organs found in wig-1 plants seems to be dependent on environmental conditions and genetic background. wig- 1 plants grown under suboptimal conditions, such as overcrowding or higher temperatures, develop fewer organs in all four whorls, sometimes resembling wild-type in organ number. wig- 1 was isolated in the Landsberg erecta (Ler) ecotype; when crossed to the Columbia (Col-2) ecotype, wig-1 mutant flowers showed greater numbers of organs, especially in basal flowers, than were found in a pure L-er
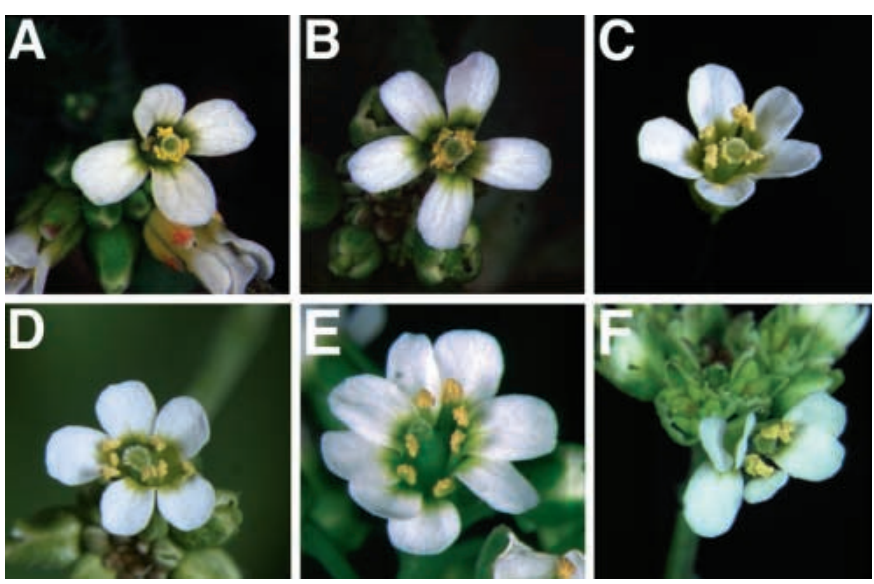

Fig. 1. Flowers of wild type and wiggum mutants. (A) Wild-type Arabidopsis flower, Landsberg erecta ecotype, with 4 sepals, 4 petals, 6 stamens and 2 carpels in stereotypical positions. (B) A wig1 mutant flower, in L-er. wig- 1 flowers have greater numbers of floral organs, especially sepals and petals. (C) A wig-2 mutant flower, in Ler. (D) A wig-3 mutant flower, in L-er. (E) wig-1 plant in a mixed Columbia (Col-2)-L-er background, with more floral organs than found in a pure L-er background. (F) wig-1 in L-er, showing petals in abnormal orientations, possibly due to abnormal growth of sepals that restrict petal growth.

background (Fig. 1E). The increase in organ number in wig-2 compared to wig-1 and wig-3 may also be due to ecotypic differences. Wild type No-0 plants, unlike L-er plants, occasionally produce flowers with 5 sepals or petals, suggesting the possibility that genes enhancing perianth organ number present in No-0 may have been carried through two crosses of wig-2 into L-er.

\section{Flower organ initiation and development in wiggum mutants}

Scanning electron microscopy (SEM) reveals that the sepals in wig flowers initiate in an aberrant manner. In wild-type flowers, 4 sepals initiate evenly spaced from each other, with 2 in lateral positions and 2 in medial positions. The medial sepals are larger and arise higher on the floral meristem than the lateral

Table 1. Mean number of wig floral organs

\begin{tabular}{|c|c|c|c|c|}
\hline Genotype & Sepals & Petals & Stamens & Carpels \\
\hline \multicolumn{5}{|c|}{ Flowers no. $1-5^{*}$} \\
\hline Ler & $4.0 \pm 0$ & $4.0 \pm 0$ & $5.8 \pm 0.6$ & $2.0 \pm 0$ \\
\hline wig-1 & $5.3 \pm 0.8$ & $5.4 \pm 0.8$ & $6.3 \pm 0.8$ & $2.4 \pm 0.5$ \\
\hline wig-2 & $6.0 \pm 1.0$ & $6.5 \pm 0.9$ & $6.4 \pm 0.8$ & $2.4 \pm 0.5$ \\
\hline wig-3 & $4.9 \pm 0.8$ & $5.4 \pm 1.0$ & $6.3 \pm 0.7$ & $2.7 \pm 0.5$ \\
\hline \multicolumn{5}{|c|}{ Flowers no. $6-10 \dagger$} \\
\hline Ler & $4.0 \pm 0$ & $4.0 \pm 0$ & $5.8 \pm 0.6$ & $2.0 \pm 0$ \\
\hline wig-1 & $4.7 \pm 0.6$ & $5.1 \pm 0.7$ & $5.6 \pm 0.6$ & $2.6 \pm 0.6$ \\
\hline wig-2 & $5.9 \pm 0.9$ & $6.2 \pm 0.9$ & $6.0 \pm 0.8$ & $2.3 \pm 0.5$ \\
\hline wig-3 & $4.8 \pm 0.8$ & $5.3 \pm 0.9$ & $6.0 \pm 0.8$ & $2.5 \pm 0.5$ \\
\hline
\end{tabular}

The average number of organs per flower is given. The standard error is indicated.

$*_{n}=60$ Ler plants, 68 wig- 1 plants, 15 wig- 2 plants and 15 wig-3 plants. $\dagger n=40$ Ler plants, 41 wig- 1 plants, 15 wig-2 plants and 15 wig-3 plants. Ler, Landsberg erecta. 
Fig. 2. Microscopic analysis of developing wild-type and wig flowers. (A) SEM of a wild-type inflorescence with very young flowers (up to stage 6). Flowers arise in a spiral pattern approximately $140^{\circ}$ from each other. On each flower, 2 large medial sepals and 2 small, lower lateral sepals are visible, and the sepals grow to enclose the bud. (B) SEM of a wig-1 inflorescence. The phyllotaxy of wig- 1 plants sometimes deviates from that seen in wild type. The sepals do not arise in a particular pattern and can vary greatly in size. They are generally shorter and thicker than wild type and fail to enclose the developing flower. (C) SEM of a wig-1 inflorescence, showing deviations in phyllotaxy and aberrant sepal initiation. The floral meristem is noticeably wider than wild type at these stages of flower development. (D) SEM of a wild-type stage 5 flower. Two of the large medial stamen primordia are visible. Two small petal primordia are visible between the sepals. Two large medial sepal primordia are more prominent and higher on the flower meristem. (E) SEM of a wig-1 flower at stage 5, with irregular stamen and petal initiation patterns. (F) CLSM of a wild-type flower at late stage 6, after sepals have enclosed the bud. (G) CLSM of a wig- 1 flower at late stage 6 . The sepals often do not elongate
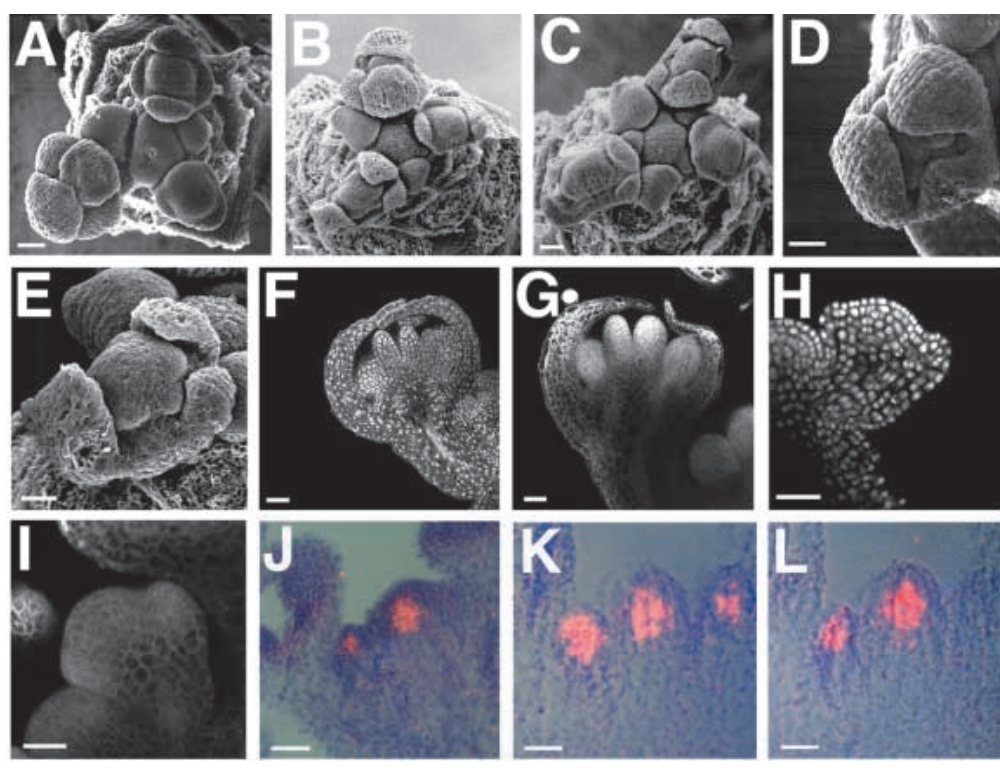

sufficiently to enclose the bud, and the flower is wider than a late stage 6 wild-type flower. $(\mathrm{H})$ CLSM of wild-type flower at mid stage 3 , when sepals have initiated. (I) CLSM of wig- 1 flower at mid stage 3, when the sepals are beginning to initiate. The flower is wider than a mid stage 3 wild-type flower. (J) Expression of CLV1 RNA in a wild-type inflorescence apex. (K-L) Expression of CLV1 RNA in wig-1 inflorescence apeces. Scale bars, A-I, $10 \mu \mathrm{m} ; \mathrm{J}-\mathrm{L}, 40 \mu \mathrm{m}$.

sepals, indicating that sepals have positional identity around the whorl (Fig. 2A, Smyth et al., 1990). In wig mutants, sepals do not arise in a predictable pattern (Fig. 2B-C), and they are often not evenly spaced from each other. They also tend to lose positional identity; that is, the size of the sepal does not necessarily depend on its position. Sepal size is variable, ranging at maturity from much smaller to somewhat larger than wild-type sepals.

In a wild-type flower, all petals are the same size and are evenly spaced from each other, arising alternately with and interior to the sepals (Smyth et al., 1990). Petals in wig plants are usually close to normal in size, but arise in positions unrelated to the positions of the sepals. There are more petals than sepals on average, and the number of petals in a particular flower shows no correlation to the number of sepals in that flower. Wild type flowers generally have 2 short lateral stamens and 4 taller medial stamens, though one or both lateral stamens are missing in about $25 \%$ of flowers (Smyth et al., 1990). In wig flowers, stamens, like the other floral organs, are not positioned in a regular manner, and their size is not necessarily related to their position, as it is in wild-type flowers (Fig. 2D,E).

Some effects on later floral organ development are observed in wig flowers. The flowers tend to open precociously, and the sepals are shorter and rougher than in wild type, and sometimes wrinkled, with surface cells enlarged (Fig. 2E). SEM and confocal laser scanning microscopy (CLSM) analysis showed that sepals in wig- 1 are shorter and thicker than wild-type sepals at a comparable developmental stage (Fig. 2A-G). Petal and stamen morphology appear fairly normal; however, petals on some flowers emerge from the flower in abnormal orientations and sometimes appear wrinkled (Fig. 1F), though these effects might have resulted from physical constraints imposed by the abnormal sepals during petal elongation. In addition, larger, compound stamens are sometimes observed, with a thicker filament and a large single anther or 2 separate anthers. The gynoecium is typically shorter than wild type at maturity, and contains fewer seeds. Thus the overall fecundity of wig mutants is reduced, though wig is both male and female fertile.

In wig mutants, the floral meristem is larger than wild type, including at stage 6 , just after petal primordia have emerged (Fig. 2F,G; all stages according to Smyth et al., 1990), and at stage 3, when sepals begin to emerge (Fig. 2H,I). At this stage, wild-type flowers are $30 \pm 3 \mu \mathrm{m}$ wide while wig- 1 flowers are $37 \pm 3 \mu \mathrm{m}$ wide (10 flowers counted per genotype). In the case of wig, the organ number alteration is most dramatic in the outer whorls, which is the area most enlarged in the meristem before organ initiation occurs.

\section{Expression of CLAVATA1 RNA in wig-1 mutants}

Like wig mutants, clavatal (clv1) mutant plants also have enlarged floral meristems (Clark et al., 1995), and CLV1 is thought to play a role in regulating cell proliferation in meristematic regions (Clark et al., 1997). Since the clvl and wig mutant phenotypes display some similarities, we investigated $C L V 1$ expression in wig mutants to see if the enlarged floral meristem phenotype was due to misexpression of the $C L V 1$ gene. The $C L V 1$ gene is expressed primarily in $\mathrm{L} 3$ of the presumptive central zone of apical and floral meristems, and downregulated of the peripheral zone from which flowers and floral organs are initiated (Clark et al., 1997; Fig. 2J). The general pattern of CLV1 expression is not substantially different in wig-l compared to wild type (Fig. 2K,L). Expression is seen in the L3 of the presumptive central zone, with no expression in the periphery of apical and floral meristems. The domain of both the expressing and nonexpressing cells, however, appears to be increased in size, suggesting that both central and peripheral zones may be larger in wig- 1 than in wild type. 


\section{Effects of wig on general plant growth and development}

Overall, wig mutant plants have a growth pattern and body plan similar to wild-type plants. There are, however, notable differences in certain aspects of growth and development. wig$l$ plants germinate less well than wild type: under controlled germination conditions (see Materials and Methods) where nearly all wild-type seeds germinate, only $64 \%$ of wig seeds germinate (Table 2). In addition, germination is delayed under ideal conditions: the median time of germination of wild-type L-er seedlings is 2 days, while in wig- 1 it is 4 days, with some wig seeds delaying germination 2 weeks or more. wig- 2 and wig-3 also affect germination rate and timing, with wig-2 showing a more dramatic effect (Table 2). Decreased and delayed germination of wig- 1 is also seen in the absence of cold treatment (stratification), which speeds germination and increases the germination rate of wild-type seeds. This decrease and delay of germination is due to zygotic effects, since plants heterozygous for wig- 1 show a lower than expected number of wig-1 homozygous progeny. However, wig-1 mature embryos appear morphologically normal.

Of wig- 1 plants that germinate, flowering (bolting) is also delayed, and rosette leaf number is slightly increased (Table 2). Maturation and senescence are also delayed, with wig- 1 life spans longer than wild type. wig-2 and wig-3 also show delayed flowering time (Table 2) and longer life span.

While the apical meristem of wig-1 plants usually appears fairly normal in size and structure, occasional fasciation of the apical meristem is observed. In addition, internode length is decreased in wig, resulting in shorter plants, and wig roots are shorter than wild-type roots at comparable ages. Phyllotaxy defects are sometimes observed as well, with the angle between flowers occasionally deviating from the approximately 140 degree angle found in wild type (Fig. 2A-C). Nevertheless, the plants are generally healthy and male and female fertile.

\section{Double mutants of wiggum and other genes controlling meristem and flower development}

\section{wig shows synergistic interactions with other mutations affecting meristem size}

Strong alleles of $c l v l$ lead to an increase in apical and floral meristem size as well as floral organ number (Fig. 3A; Leyser and Furner, 1992; Clark et al., 1993), and mutations in the clv3 gene have similar effects (Fig. 3E; Alvarez and Smyth, 1994; Clark et al., 1995). The double mutant of wig-1 and clv1-4, a strong $c l v 1$ allele, shows an additive phenotype in early flowers, with more enlarged apical meristems and more of each type of floral organ (Fig. 3B). Later in development the double mutant

Table 2. wig germination frequency and flowering time

\begin{tabular}{lccc}
\hline Genotype & \% Germination* & Days to bolt $\dagger$ & Rosette leaf no. $\dagger$ \\
\hline Ler & 99 & $22.4 \pm 1.5$ & $8.6 \pm 0.8$ \\
wig-1 & 64 & $30.2 \pm 1.5$ & $9.1 \pm 1.1$ \\
wig-2 & 59 & $38.1 \pm 2.3$ & $14.9 \pm 3.0$ \\
wig-3 & 64 & $34.2 \pm 2.4$ & $9.4 \pm 1.7$ \\
& \\
& *Percentage germination of 200 seeds scored per allele. \\
& \\
& Ler Mean and standard error of 30 plants scored per allele. \\
& \\
\hline
\end{tabular}

phenotype becomes much more severe: the apical meristem is greatly disrupted, often increasing to a diameter of $1 \mathrm{~cm}$ or more, and then either differentiates into tissue that resembles stigmatic tissue (Fig. 3C) or remains undifferentiated overproliferating meristem tissue (Fig. 3D). In addition, the plants are shorter and have greatly reduced internode length and severe phyllotaxy disruptions, and fewer flowers are formed compared to wild type or either single mutant. Later flowers sometimes have reduced numbers of organs compared to wild type, indicating a severe disruption in floral meristem function. This is also seen by the lack of determinacy in wig-1 clv1-4 flowers, with undifferentiated floral meristem tissue sometimes emerging from the carpels. Because of this, wig- $1 \mathrm{clvl}-4$ plants are much less fertile than wild type or either single mutant.

The double mutant of $c l v 3-2$ (a strong $c l v 3$ allele) with wig1 shows similar effects: the phenotype is additive in the earliest flowers (Fig. 3F), but soon the apical meristem greatly enlarges (Fig. 3G,H), and later flowers have reduced organ number and loss of determinacy resulting in undifferentiated floral meristem tissue emerging from the carpels (Fig. $3 \mathrm{H})$. The double mutant combination of wig- 1 with the intermediate clvl-1 allele does not have as severe a phenotype as with the strong $c l v 1$ and $\operatorname{clv} 3$ alleles, with no enhancement of apical meristem defects compared to either single mutant. The $c l v 2-1$ mutant resembles weak clvl alleles in its severity in organ number increase, affecting only the carpel number (Koornneef et al., 1983); double mutants of wig- 1 with clv2-1 appear additive.

Mutations in fas 2 cause changes in floral organ number, producing flowers with slightly more sepals and slightly fewer petals than wild type (Fig. 3I; Leyser and Furner, 1992). In addition, fas 2 plants show some similarities to wig in their vegetative growth: fas 2 mutants flower about 1 week later than wild type, and produce an extra leaf before flowering, and the leaves are slightly smaller and darker green. The fas 2 wig- 1 double mutant shows a synergistic interaction (Fig. 3J): sepal, petal and stamen number are all reduced compared to wild type or either single mutant (Table 3). In addition, the plants take longer to flower than either single mutant alone, and are much less fertile. fas 1 shows a similar but less severe change in organ number compared to fas2 (Leyser and Furner, 1992). The fas 1 wig- 1 double mutant appears additive with respect to organ number and plant growth.

\section{wig is additive with perianthia and superman}

Mutations in the PERIANTHIA gene lead to a similar change in organ number as do mutations in WIG. Sepal, petal, and stamen number all average 5 in number, and 2 carpels are usually present (Fig. 3K). The mechanism of PAN gene activity appears different, however, since no floral meristem size change is detected in pan mutants, and organs arise in a regular, predictable pattern (Running and Meyerowitz, 1996). The wig -1 pan-1 double mutant phenotype is additive (Fig. 3L). Flowers average 6 sepals and petals, along with 6-7 stamens and 2-3 carpels (Table 3). This suggests that wig and pan act in separate processes in the determination of organ number.

The SUPERMAN (SUP; also called FLO10) gene affects the position of the boundary between the third and fourth whorl, and sup mutants show increased stamen number at the expense of carpel tissue (Fig. 3M; Schultz et al., 1991; Bowman et al., 1992; Sakai et al., 1995). The wig-1 sup-1 double mutant phenotype (Fig. $3 \mathrm{~N}$ ) is additive with respect to sepal, petal and 
Table 3. Mean number of floral organs in wig double mutants

\begin{tabular}{llllrl}
\hline Genotype & $n$ & Sepals & Petals & Stamens & Carpels \\
\hline Flowers no. 1-5 & & & & & \\
$\quad$ fas 2 & 50 & $4.6 \pm 0.8$ & $4.0 \pm 0.6$ & $5.7 \pm 0.7$ & $2.3 \pm 0.3$ \\
$\quad$ wig-1 fas 2 & 35 & $4.0 \pm 0.7$ & $3.1 \pm 0.9$ & $5.1 \pm 0.9$ & $2.4 \pm 0.6$ \\
$\quad$ pan-1 & 49 & $5.1 \pm 0.7$ & $4.8 \pm 0.8$ & $5.6 \pm 0.6$ & $2.0 \pm 0.0$ \\
wig-1 pan-1 & 50 & $5.9 \pm 0.6$ & $5.8 \pm 0.6$ & $6.7 \pm 0.8$ & $2.7 \pm 0.5$ \\
sup-1 & 25 & $4.0 \pm 0$ & $4.0 \pm 0$ & $12.4 \pm 2.3$ & $1.2 \pm 0.7$ \\
wig-1 sup-1 & 20 & $4.8 \pm 0.8$ & $5.1 \pm 0.9$ & $28.6 \pm 5.0$ & $0.9 \pm 0.7$ \\
& & & & & \\
Flowers no. 6-10 & & & & & \\
fas2 & 50 & $4.3 \pm 0.6$ & $4.0 \pm 0.6$ & $5.4 \pm 0.7$ & $2.2 \pm 0.4$ \\
$\quad$ wig-1 fas-2 & 34 & $3.8 \pm 0.7$ & $3.0 \pm 1.1$ & $4.4 \pm 1.4$ & $2.0 \pm 0.7$ \\
pan-1 & 49 & $4.9 \pm 0.6$ & $5.0 \pm 0.8$ & $5.4 \pm 0.8$ & $2.0 \pm 0.0$ \\
wig-1 pan-1 & 50 & $5.5 \pm 0.6$ & $5.6 \pm 0.6$ & $6.2 \pm 0.9$ & $2.3 \pm 0.5$ \\
sup-1 & 25 & $4.0 \pm 0.0$ & $4.0 \pm 0.0$ & $10.0 \pm 1.3$ & $1.3 \pm 0.7$ \\
wig-1 sup-1 & 20 & $4.7 \pm 0.9$ & $4.9 \pm 0.9$ & $26.5 \pm 4.9$ & $0.7 \pm 0.5$
\end{tabular}

The average number of organs per flower is given. The standard error is indicated.

$n$, number of flowers analyzed.

carpel number. However, stamen number in the double mutant is significantly increased over that in either single mutant (Table 3), revealing that wiggum may play a role in controlling cell proliferation between as well as within floral whorls.

wig is additive with the floral homeotic genes

Several flower mutants are known to show a homeotic conversion from one organ type to another type found elsewhere in the flower (reviewed in Weigel and Meyerowitz, 1994; Yanofsky, 1995). These fall into 3 classes, with each class affecting the identity of organs in 2 adjacent whorls. The class A genes include APETALAl (API), which also affects floral meristem identity, and APETALA2 (AP2). Mutations in both genes cause the conversion of sepals to leaf-like organs or carpels, and the partial conversion of petals to stamens in some alleles. Double mutant combinations of wig- 1 with ap2-2 (a strong $a p 2$ allele) are additive, with organ number intermediate between the two single mutants, and organ identity the same as found in ap2-2. Similar additive effects are seen in the double mutant wig-1 ap 1-1 (a strong apl allele). The class B genes are APETALA3 (AP3) and PISTILLATA (PI), mutations in either of which cause a conversion of petals to sepals and of stamens to carpels. wig- 1 in combination with either ap3-3 (a strong ap 3 allele) or $p i-1$ (a strong $p i$ allele) is additive, with organ number per whorl characteristic of wig-1 single mutants, and organ identity characteristic of ap3-3 or pi-1. The class $\mathrm{C}$ gene is AGAMOUS (AG), which when mutated causes the conversion of stamens to petals and carpels to sepals, as well as the generation of additional whorls in the center of the flower (Fig. 30). The double mutant wig- 1 ag-3 (a strong ag allele) also has an additive phenotype. The organ number in the first and second whorl is increased as in wig-1, with the organ type the same as seen in the ag-3 mutant (Fig. 3P). Thus wig acts independently of the floral homeotic genes in determining flower pattern.

wig shows synergistic interactions with mutations affecting floral meristem identity

The API and LEAFY (LFY) genes have been shown to affect floral meristem identity (reviewed by Weigel, 1995). Mutation in these genes leads to a partial conversion of flowers into shoot structures. Combinations of mutations in genes affecting floral meristem structure with those affecting floral meristem identity, particulary lfy, have been shown to have a severe synergistic effect, increasing the severity of both the disruption of floral meristem structure and loss of flower meristem identity (Clark et al., 1993, 1994; Clark and Meyerowitz, 1994; Levin and Meyerowitz, 1995). Similar interactions are seen with meristem structure mutants in combination with three other mutants having weaker effects on floral meristem identity: unusual floral organs (ufo; Levin and Meyerowitz, 1995; Wilkinson and Haughn, 1995), hanaba taranu (han; H. Sakai, M. P. R. and E. M. M., unpublished) and filamentous flower (fil, also called fl54, Komaki et al., 1988; Okada and Shimura, 1994; Clark et al., 1994; Clark and Meyerowitz, 1994).

wig mutants also show synergistic interactions in double mutant combinations with lfy, ufo, han, and fil, with some differences from those seen in previous studies with other mutants. The most severe phenotype is observed in the wig- 1 lfy-6 mutant (Fig. 4A,B). The primary apical meristem terminates prematurely in a carpel-like structure after producing a few reduced flowers and several filamentous structures. Secondary inflorescences give rise only to filaments before terminating. Double mutants of wig- 1 with $u f o-2$ also produce reduced flowers with fewer organs or flowers completely replaced by filaments (Fig. 4C,D). The shoots terminate in carpelloid structures, much earlier than in ufo alone. Flower development is not as disrupted as in wig lfy plants, since many flowers with flower parts, predominantly sepals and carpels, are formed. Similar results are seen in double mutants of wig fil. fil single mutant flowers have smaller and fewer organs, and occasional filaments are initiated from the inflorescence (Fig. 4E; Komaki et al., 1988; Okada and Shimura, 1994). The double mutant of fil with wig shows more filaments in place of flowers, fewer organs in flowers that do form, and earlier termination of the main stem, compared to either single mutant (Fig. 4F). Mutations in han lead to flowers with fewer organs, especially petals and stamens (Fig. 4G,H. Sakai, M. P. R. and E. M. M., unpublished); in combination with wig-1, filaments are occasionally formed in place of flowers (Fig. 4H). In general, double homozygotes of wig with these mutants are less severe in phenotype than strong $c l v$ allele double mutants with these genes, since wig ufo, wig han and wig fil still produce flowers.

\section{wig shows synergistic interactions with several other mutations affecting flower development}

leunig (lug) mutant plants show a variety of defects, including slightly later flowering, narrow leaves and floral organs, and ectopic homeotic gene expression (Fig. 4I; Liu and Meyerowitz, 1995). The double mutants lug lfy and lug ufo also have reduced flowers and filamentous structures (Levin and Meyerowitz, 1995). Double mutants of wig- 1 and lug-3, a strong lug allele, do not produce filaments, but flowers often lack petals and have fewer organs than in lug-3 alone (Fig. 4J).

Mutations in the SNOWBALL gene lead to flowers with narrow organs, with 4-5 sepals and 3-5 petals in most flowers (Fig. 4K; R. W. Williams, M. P. R. and E. M. M., unpublished). The sno wig-1 double mutant shows a severe disruption in floral and apical meristem structure. The main stem initiates 
Fig. 3. Double mutants of wig with other genes affecting floral organ number. (A) clv1-4 mutant flowers, which have extra organs in all 4 whorls, especially carpels. (B) The first flowers of a wig-1 clv1-4 double mutant, showing an additive phenotype with respect to floral organ number (more organs in each whorl compared to either single mutant). (C) wig-1 clv1-4 apex at a later stage, with the apical meristem overproliferating and differentiating into stigmatic tissue. Flowers have fewer organs than in either single mutant, seen here in the reduced number of carpels in the gynoecium. (D) Another wig- 1 clvl-4 apex, with massive overproliferation of apparently undifferentiated meristem tissue. (E) clv3-2 flowers, which display a phenotype very similar to $c l v 1-4$. (F) Early flowers of a wig-1 clv3-2 double mutant plant, showing an additive phenotype with respect to organ number. $(G)$ An older wig-1 clv3-2 plant, with overproliferation of the apical meristem. (H) Another older wig-1 clv3-2 plant with overproliferation of both the apical meristem and undifferentiated floral meristem cells that have grown out of carpels. (I) A fas 2 mutant, which has flowers with 4-5 sepals and 3-5 petals. (J) wig- 1 fas 2 double mutant, which has flowers with fewer organs, especially petals and stamens, compared to wig- 1 and fas 2 single mutants. (K) A pan-1 flower, which has floral organs similar in number to wig-1 mutants, including extra sepals and petals and occasionally extra carpels. (L) A wig-1 pan-1 flower, which shows an additive organ number phenotype. (M) A sup- 1 flower, with more stamens and a reduced gynoecium compared to wild
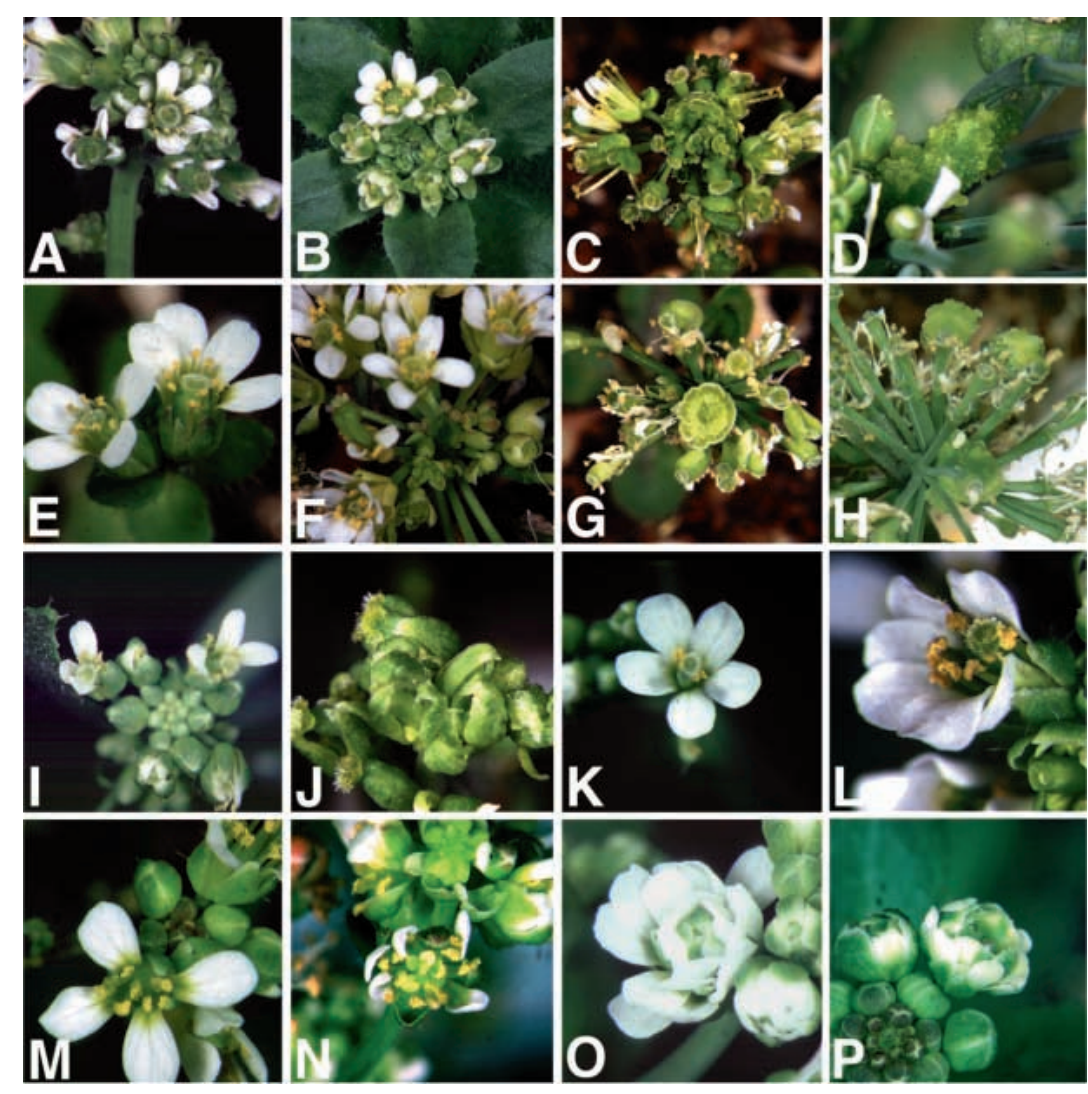
type. (N) A wig-1 sup-1 flower, with an additive phenotype with respect to organ number. $(\mathrm{O})$ An ag-3 mutant flower, in which stamens are converted to petals and carpels converted to sepals, with additional whorls of petals and sepals forming interior to the fourth whorl. (P) A wig-1 ag-3 flower, which shows an additive phenotype: homeotic conversions of floral organ type and extra whorls as seen in ag-3, with extra organs in each whorl as in wig-1.

numerous filaments or sepal-like organs and occasionally structures recognizable as incomplete flowers, missing most floral organs (Fig. 4L).

\section{DISCUSSION}

We have studied the effects of mutations in the WIGGUM gene on Arabidopsis development. wig mutant flowers form more floral organs than wild type, particularly sepals and petals, a phenotype correlated with an increase in floral meristem width. wig mutants also have some vegetative growth defects, indicating that $W I G$ is likely to be active in all stages of plant development. Many of the visible pleiotropic phenotypes, including reduced germination, slower growth, and faulty initiation of floral organs leading to abnormal sepal shape and position, may be due primarily to underlying defects in the apical and floral meristems of wig mutant plants.

\section{Similarities and differences between wiggum and previously described mutants affecting flower development}

Certain aspects of the wiggum mutant phenotype resemble those reported for other Arabidopsis mutants. In terms of floral organ number, the mutant most closely resembling wig is pan (Running and Meyerowitz, 1996), which also shows an increase in sepal and petal number without dramatic increases in stamen or carpel number. In most other ways, though, wig resembles mutants that have been shown to affect apical and floral meristem structure.

The organ number increases seen in pan and wig flowers seem to be due to different mechanisms. pan mutations do not cause disruptions in overall apical and floral meristem structure, and changes in organ number in pan mutants are presumably caused by changes in the spacing and position of floral organs (Running and Meyerowitz, 1996). wig mutations, on the other hand, cause the formation of floral meristems wider in girth than wild type, which allows for the formation of more organs in the outer whorls. This is analogous to the mechanism proposed for the increase in organ number seen in clv1 and clv3 mutants (Clark et al., 1993, 1995; Alvarez and Smyth, 1994). In clv plants the floral meristem is taller than wild type, leading to extra organs in the inner whorls as well as extra whorls, and alleles with more severe organ number defects have a greater size increase in the floral meristem. The fact that organ number changes in wig, $c l v 1$, and $c l v 3$ can be correlated with specific changes in floral meristem size at the time of organ initiation indicates that one mechanism flowering plants use to regulate floral organ number is the regulation of floral meristem size.

wig mutants do not initiate organs in a consistent or predictable pattern, which is also the case for $c l v 1$ and $c l v 3$ (Clark et al., 1993, 1995). In wild-type flowers, organs arise in stereotypical positions, and in pan flowers certain aspects of 
wild-type organ position are maintained, like the presence of an adaxial sepal and the dependence of petal position on sepal position. Sepals of wig mutant flowers can arise in any position around the floral meristem, with petal position not necessarily showing any relationship to sepal position. Also, positional differences in floral organs seem to be lost in wig and clv mutants; for instance, the size of an organ is not strictly dependent on where it arises, as they are in wild type and pan. These results suggest that disruptions of floral meristem structure, as seen in wig and clv, lead to defects in organ positioning and positional identity.

Unlike pan and $c l v$ plants, mutant wig plants show a number of abnormalities outside of the apical and floral meristem, including lower germination rate, slower germination, longer time to bolting, decreased internode length, and decreased root length. This suggests that WIG is required in areas outside the apical and floral meristem, in wider domains than $P A N$ and $C L V$. Other mutations affecting meristem structure have pleiotropic effects as well: the fas $2, t s l, l u g$, and rev mutations cause defects in vegetative leaf and floral organ development, and later flowering (Leyser and Furner, 1992; Roe et al., 1993; Liu and Meyerowitz, 1995; Talbert et al., 1995).

\section{Genetic interactions between wig and other Arabidopsis mutants are consistent with a role for wig in control of meristematic cell division}

Studies of genetic interactions between wig and previously described mutants give results consistent with a role for WIG in the control of floral and apical meristem structure deduced by phenotypic studies of wig single mutants. Synergistic interactions are seen with mutants that have been shown to play a role in the same or similar processes, while additive interactions are seen with mutants that do not disrupt meristem structure.

The most striking example of genetic synergy is seen in interactions between wig and strong $c l v 1$ and $c l v 3$ alleles. Both $C L V 1$ and $C L V 3$ have been implicated in the control of apical and floral meristem size, and mutations in either gene lead to an overproliferation of cells in these regions (Leyser and Furner, 1992; Clark et al., 1993, 1995; Alvarez and Smyth, 1994), although the changes induced by $c l v$ mutations are distinct from those seen in wig. CLV1 and CLV3 have similar phenotypes and appear to act in the same pathway, since double mutants of strong clv 1 and $c l v 3$ alleles resemble both single mutants (Clark et al., 1995). Double mutants of strong clv alleles with wig show exaggerated proliferation of cells in older apical and floral meristems, seemingly due to a much greater loss of control of cell proliferation than that seen in either single mutant. However, the apical meristem on younger double mutant plants does not show gross morphological defects, and the early flowers show an additive phenotype. This phenotype, along with the absence of any epistatic interactions, indicates that $W I G$ acts in a separate pathway from $C L V 1$ and $C L V 3$ in control of cell division in apical and floral meristems. Expression of CLV1 in wig-1 mutants is not substantially different from that seen in wild type, supporting the notion that $C L V 1$ function is important in restricting cell proliferation in wig-1.

The FAS2 gene has also been shown to play a role in the control of apical meristem structure, and shows a slight change in floral organ number (Leyser and Furner, 1992). wig fas 2 double mutant plants have flowers with fewer organs than either single mutant, suggesting that $W I G$ is involved in a similar process but in a different pathway than FAS2. In contrast, wig shows an additive interaction with pan, a mutation that affects floral organ number without affecting meristem size or structure. This supports the idea that PAN and $W I G$ control floral patterning via two separable mechanisms, $W I G$ by controlling, in the floral meristem, the amount of cell proliferation relative to withdrawal of cells into developing
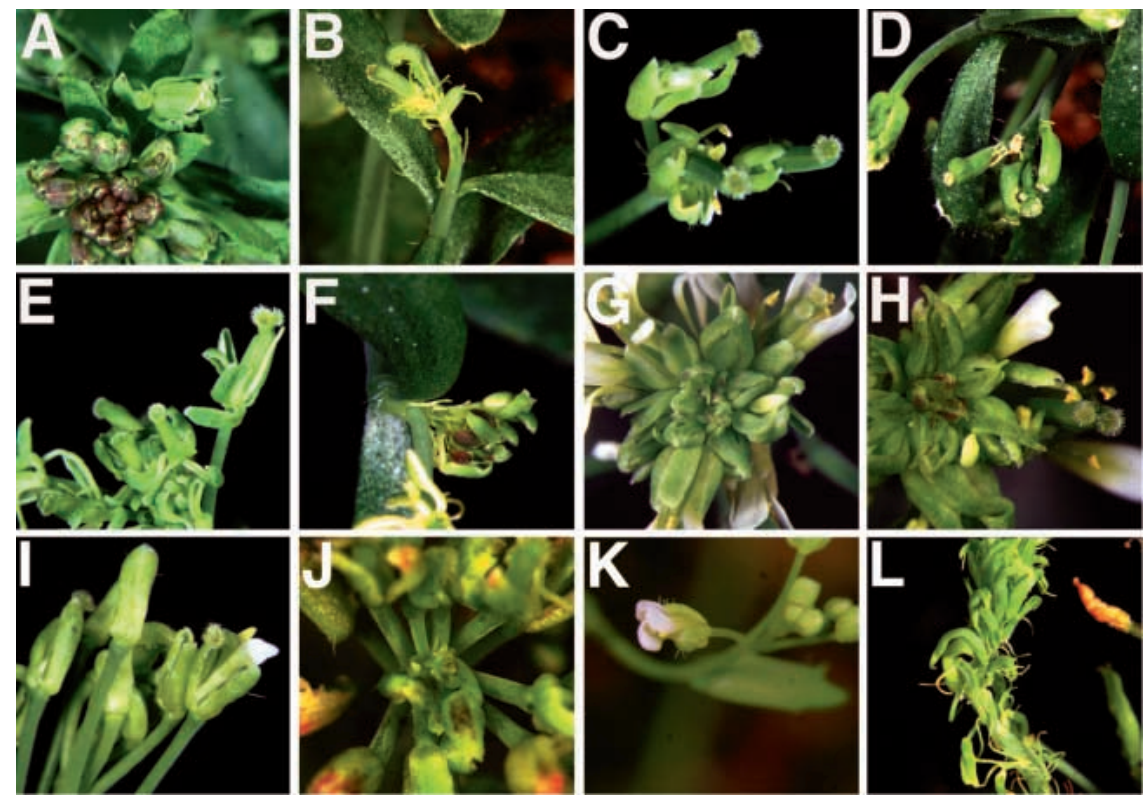

Fig. 4. Enhancement of meristem defects of wig in combination with other mutations affecting flower development. (A) A lfy-6 mutant apex. Ify mutants lead to a partial conversion of flowers into shoot structures, accompanied by loss of some floral organ identity. (B) A wig-1 lfy-6 double mutant, which shows filamentous structures in place of flowers, and early termination of the apical meristem in carpel structures. (C) ufo-2 mutant inflorescence, which is also defective in floral meristem identity, though not as severely as in lfy mutants. (D) wig- 1 ufo- 2 double mutant plants show early termination of the main stem into carpels, and some flowers are replaced by filaments. (E) fil mutant flowers, which have defects in organ number, size and position. (F) A wig-1 fil double mutant apex, with many flowers reduced in organ number or replaced by filaments. The inflorescence displays both phyllotaxy defects and early termination of the apical meristem. (G) han-1 mutant flowers, showing reduced organ number, especially in petal and stamen whorls. (H) wig-1 han-1 double mutant apex, with some flowers replaced by filaments. (I) lug-3 mutant flowers, in which floral organs are narrower. (J) wig-1 lug-3 double mutant flowers, which have reduced organ numbers, though no filament structures are seen. (K) sno mutant flowers, which have extra sepals and 3-5 petals. (L) A wig-1 sno double mutant inflorescence, which produces many filaments and occasionally flowers with dramatically reduced organ number. 
organs, and PAN by controlling the distance between organ initiation sites. In addition, ag, ap1, ap2, ap3 and pi, mutations affecting floral organ identity, are additive in combination with wig, indicating that $W I G$ acts independently of the floral homeotic genes in establishing flower pattern. CLVI also acts independently of PAN and the homeotic genes (Clark et al., 1993), which is consistent with $C L V$ genes acting in the same process as $W I G$, distinct from genes that do not affect meristem structure. wig- 1 also shows largely additive phenotypes with sup-1, though the number of stamens in the double mutant is dramatically increased, indicating a role for $W I G$ in restricting proliferation in sup- 1 .

\section{Enhancement of meristem defects in wig by lfy and other mutations causing defects in floral meristem identity}

Previous studies have shown that certain combinations of meristem mutations show a dramatically enhanced phenotype, leading to severe disruptions in apical meristem function (Clark et al., 1993, 1994; Clark and Meyerowitz, 1994; Levin and Meyerowitz, 1995). Defects include extreme phyllotaxy abnormalities and failure to initiate complete flowers, with filamentous structures formed in their place. Most commonly, these filamentous structures are seen in combinations of mutations defective in apical meristem structure (such as $c l v l$, clv3, and han) and mutations affecting floral meristem identity (such as lfy, ufo, and fil), though not all double mutants show this phenotype. The wig mutation also causes filamentous structure formation and other apical meristem defects in combination with most of these mutations. The most severe defects are seen with wig and strong lfy mutants, which rarely form flowers, while wig with $u f o$, fil, or han mutants show occasional filament structures. The sno mutant also seems to fall into the filament producing class, with a particularly severe effect in combination with wig.

The fact that certain wig double mutant combinations lead to filament structures is surprising, given that lfy, ufo and fil show single mutant phenotypes seemingly unrelated to those seen in wig. It is possible that these genes also play a role in maintenance of meristem structure, and their role becomes more important in the background of mutations that also disrupt meristem structure, such as wig, clv1 and clv3. This implies a partial redundancy among these genes, and suggests a threshold of meristem maintenance activity, below which meristems become increasingly abnormal. This idea is supported by the fact that double mutant combinations of wig and weaker mutant alleles of other genes do not show a severe disruption of meristem activity, as does wig in combinations with stronger alleles. For instance, while wig-1 clv1-4 plants have greatly disrupted meristems, wig-1 clv1-1 plants do not, nor do wig-1 clv2-1 plants. Also, while wig interacts with many of the same mutations as $c l v l$, it does so in a less severe fashion, perhaps reflecting the more severe phenotypes found in clvl single mutants compared to wig. Similar threshold effects are seen in clv1 apl-1 double mutants, with clv1-4 showing a synergistic effect, and clv $1-1$ showing an essentially additive phenotype with ap1-1 (Clark et al., 1993). There are indications this threshold increases as the shoot gets older, since meristem disruption in the double mutants becomes progressively worse in all cases, especially notable in the wig clv doubles.

\section{Model of wig function in apical and floral meristems}

The phenotype of wig plants suggests at least two models predicting the mechanism of action and expression pattern of $W I G$. Both are based on the hypothesis that WIG is responsible for controlling cell proliferation relative to cell differentiation in certain regions of the floral meristem. Traditional histological studies have divided apical and floral meristems into three zones: the central zone $(\mathrm{CZ})$, a region of less mitotically active cells in the center of the meristem, the peripheral zone (PZ), a more densely staining region of more actively dividing cells surrounding the central zone and giving rise to leaf and flower primordia, and the rib meristem, below the $\mathrm{CZ}$ (reviewed by Meyerowitz, 1997). WIG may be responsible for negatively regulating cell proliferation or increasing the rate of cell differentiation in the PZ, and, in the absence of WIG function, the number of cells in this region increases relative to wild type, leading to meristem expansion and occasional fasciation.

Apical and floral meristems have another level of organization, being composed of three cell layers termed L1, L2, and L3. Both L1 and L2 are single layers of cells, with L1 cells forming the epidermal layer, and L2 cells immediately below. Cells in both layers divide almost exclusively anticlinally, and are clonally distinct. The remaining cells are found in the L3, or corpus, where cells divide in many different planes (reviewed by Meyerowitz, 1997). It is possible that WIG is responsible for limiting cell proliferation in the L1 and L2 layers, and when this control is lost, the resulting increases in cell division lead to a widening of the floral meristem, because of the increased number of anticlinal cell divisions relative to cell divisions in different planes in the meristem. Cell communication between different cell layers, presumably carried out by a mechanism unrelated to $W I G$, would keep the overall meristem structure intact.

These models are complementary to models proposed for CLVI action (Clark et al., 1993, 1997). CLVI is also required for limiting cell division relative to cellular differentiation in the apical and floral meristems, but seemingly by a different mechanism than WIG, since its mutant phenotype is distinct: a much larger increase in height than in width. It has been proposed that $C L V 1$ is responsible for controlling cell proliferation in the $\mathrm{CZ}$, such that when $C L V 1$ is mutant the $\mathrm{CZ}$ is increased in size (Clark et al., 1993). An alternate model is that $C L V 1$ is required for restricting cell division in the L3 layer, such that in clvl mutants, the ratio of periclinal to anticlinal cell divisions increases, leading to a greater meristem height. The latter model is consistent with the expression pattern of CLVI RNA, which is found in the L3 layer, but not in the L1 and likely not in the L2 (Clark et al., 1997). Both models help explain the dramatic phenotype seen in double mutant combinations of $c l v 1$ and wig. Both single mutant phenotypes are less severe, since cell division control in one area of the meristem is still intact, and cell-cell communication between different layers of the meristem may help limit the total number of cells in the meristem. However, if cell division control in most or all of the meristem is disrupted, overproliferation of the entire meristem may be more likely.

We thank Detlef Weigel for providing the wig- 1 mutant, and Adam Readhead and Jane Wei for assistance in the mapping of WIG. We thank Pat Koen for assistance with the scanning electron microscope, 
and Xuemei Chen, Jian Hua, Steven Jacobsen, Carolyn Ohno, Preeya Puangsomlee, Jose-Luis Reichmann, Robert Sablowski, Robert Williams, and Eva Ziegelhoffer for comments on the manuscript. This work was supported by NSF-MCB grant no. 9603821 to E. M. M. and NIH postdoctoral grant no. GM17868 to J. C. F. M. P. R was a Howard Hughes Predoctoral Fellow. This article is dedicated to the memory of Rebecca Rothenberg.

\section{REFERENCES}

Alvarez, J. and Smyth, D. R. (1994). Flower development in clavata3, a mutation that produces enlarged floral meristems. In Arabidopsis: An Atlas of Morphology and Development. (ed. J. Bowman), pp. 254-257. New York: Springer-Verlag.

Barton, M. K. and Poethig, R. S. (1993). Formation of the shoot apical meristem in Arabidopsis thaliana: an analysis of development in the wildtype and in the SHOOT MERISTEMLESS mutant. Development 119, 823831.

Bell, C. J. and Ecker, J. R. (1994). Assignment of 30 microsatellite loci to the linkage map of Arabidopsis. Genomics 19, 137-144.

Bowman, J. L., Smyth, D. R. and Meyerowitz, E.M. (1989). Genes directing flower development in Arabidopsis. Plant Cell 1, 37-52.

Bowman, J. L., Sakai, H., Jack, T., Weigel, D., Mayer, U. and Meyerowitz, E. M. (1992). SUPERMAN, a regulator of floral homeotic genes in Arabidopsis. Development 114, 599-615.

Clark, S. E., Jacobsen, S. E., Levin, J. Z.,and Meyerowitz, E. M. (1996). The CLAVATA and SHOOT MERISTEMLESS loci competitively regulate meristem activity in Arabidopsis. Development 122, 1567-1575.

Clark, S. E. and Meyerowitz, E. M. (1994). Arabidopsis flower development In Arabidopsis (ed. E. M. Meyerowitz and C. R. Somerville), pp. 435-466. Cold Spring Harbor, NY: Cold Spring Harbor Laboratory Press.

Clark, S. E., Running, M. P. and Meyerowitz, E. M. (1993). CLAVATA1, a regulator of meristem and flower development in Arabidopsis. Development 119, 397-418

Clark, S. E., Running, M. P. and Meyerowitz, E. M. (1995). CLAVATA3 is a regulator of shoot and floral meristem development affecting the same processes as CLAVATA1. Development 121, 2057-2067.

Clark, S. E., Sakai, H. S. and Meyerowitz, E. M. (1994). Inflorescence development in clavata mutants. In Arabidopsis: an Atlas of Morphology and Development (ed. J. Bowman), pp. 214-215. New York: SpringerVerlag.

Clark, S. E., Williams, R. W. and Meyerowitz, E. M. (1997). The CLAVATA 1 gene encodes a putative receptor kinase that controlls shoot and floral meristem size in Arabidopsis. Cell 89, 575-585.

Crone, W. and Lord, E. M. (1993). Flower development in the organ number mutant clavatal-1 of Arabidopsis thaliana (Brassicaceae). Am. J. Bot. 80, 1419-1426.

Elliott, R. C., Betzner, A. S., Huttner, E., Oakes, M. P., Tucker, W. Q. J., Gerentes, D., Perez, P. and Smyth, D.R. (1996). AINTEGUMENTA, an APETALA2-like gene of Arabidopsis with pleiotropic roles in ovule development and floral organ growth. Plant Cell 8, 155-168.

Endrizzi, K, Moussian, B., Haecker, A., Levin, J. Z. and Laux, T. (1996). The SHOOTMERISTEMLESS gene is required for maintenance of undifferentiated cells in Arabidopsis shoot and floral meristems and acts at a different regulatory level than the meristem genes WUSCHEL and ZWILLE. Plant J. 10, 967-979.

Feldmann, K. A. (1992). T-DNA insertion mutagenesis in Arabidopsis: seed infection/transformation. In Methods in Arabidopsis Research (ed. C. Koncz, N.-H. Chua, and J. Schell), pp. 274-289. Singapore: World Scientific.
Klucher, K. M., Chow, H., Reiser, L. and Fischer, R. L. (1996). The AINTEGUMENTA gene of Arabidopsis required for ovule and female gametophyte development is related to the floral homeotic gene APETALA2. Plant Cell 8, 137-153.

Komaki, M. K., Okada, K., Nishino, E. and Shimura, Y. (1988). Isolation and characterization of novel mutants of Arabidopsis thaliana defective in flower development. Development 104, 195-203.

Koornneef, M., van Eden, J., Hanhart, C. J., Stam, P., Braaksma, F. J. and Feenstra, W. J. (1983). Linkage map of Arabidopsis thaliana. J. Hered. 74, 265-272.

Laux, T., Meyer, K. F. X., Berger, K. and Jurgens, G. (1996). The WUSCHEL gene is required for shoot and floral meristem integrity in Arabidopsis. Development 122, 87-96.

Levin, J. Z. and Meyerowitz, E. M. (1995). UFO: an Arabidopsis gene involved in both floral meristem and floral organ development. Plant Cell 7, 529-548.

Leyser, H. M. O. and Furner, I. J. (1992). Characterization of three shoot apical meristem mutants of Arabidopsis thaliana. Development 116, $397-$ 403

Liu, Z. and Meyerowitz, E. M. (1995). LEUNIG regulates AGAMOUS expression in Arabidopsis flowers. Development 121, 975-991.

McConnell, J. R. and Barton, M. K. (1995). Effect of mutations in the pinhead gene of Arabidopsis on the formation of shoot apical meristems. Dev. Genet. 16, 358-366.

Meyerowitz, E. M. (1997). Genetic control of cell division patterns in developing plants. Cell 88, 209-308.

Okada, K. and Shimura, Y. (1994). Genetic analyses of signaling in flower development using Arabidopsis. Plant Mol. B. 26, 1357-1377.

Roe, J. L., Nemhauser, J. L. and Zambryski, P. C. (1997). TOUSLED participates in apical tissue formation during gynoecium development in Arabidopsis. Plant Cell 9, 335-353.

Roe, J. L., Rivin, C. J., Sessions, R. A., Feldmann, K. A. and Zambryski, P. C. (1993). The Tousled gene in A. thaliana encodes a protein kinase homolog that is required for leaf and flower development. Cell 75, 938-950.

Running, M. P., Clark, S. E. and Meyerowitz, E. M. (1995). Confocal microscopy of the shoot apex. In Methods in Cell Biology, Vol. 49 (ed. D W. Galbraith, D. P. Bourque, and H. J. Bohnert), pp. 217-229. San Diego: Academic Press.

Running, M. P. and Meyerowitz, E. M. (1996). Mutations in the PERIANTHIA gene of Arabidopsis specifically alter floral organ number and initiation pattern. Development 122, 1261-1269.

Sakai, H., Medrano, L. and Meyerowitz, E. M. (1995). Arabidopsis floral whorl boundary maintenance by SUPERMAN. Nature 378, 199-203.

Schultz, E. A., Pickett, F. B. and Haughn, G. W. (1991). The FLO10 gene product regulates the expression domain of homeotic genes AP3 and PI in Arabidopsis flowers. Plant Cell 3, 1221-1227.

Smyth, D. R., Bowman, J. L. and Meyerowitz, E. M. (1990). Early flower development in Arabidopsis. Plant Cell 2, 755-767.

Talbert, P. B., Adler, H. T., Parks, D. W. and Comai, L. (1995). The REVOLUTA gene is necessary for apical meristem development and for limiting cell divisions in the leaves and stems of Arabidopsis thaliana. Development 121, 2723-2735.

Weigel, D. and Meyerowitz, E.M. (1994). The ABCs of floral homeotic genes. Cell 78, 203-209.

Weigel, D. (1995). The genetics of flower development- from floral induction to ovule morphogenesis. Annu. Rev. Genet. 29, 19-39.

Wilkinson, M. D. and Haughn, G. W. (1995). UNUSUAL FLORAL ORGANS controls meristem identity and organ primordia fate in Arabidopsis. Plant Cell 7, 1485-1499.

Yanofsky, M. F. (1995). Floral meristems to floral organs: genes controlling early events in Arabidopsis flower development. Annu. Rev. Plant Physiol. Plant Mol. Biol. 46, 167-188. 